\title{
Immunohistochemical Expression of p53 and p63 in Adenomas and Carcinomas of Canine Mammary Glands
}

\author{
Erika M. Terra ${ }^{1}$, Marcela M. M. P. Rodrigues ${ }^{2}$, Geórgia M. Magalhães ${ }^{3}$, Mirela T. Costa ${ }^{1}$, \\ Renée L. Amorim², Noeme S. Rocha ${ }^{2}$ \\ ${ }^{1}$ Veterinary Clinics and Surgery Department, Sao Paulo State University, Sao Paulo, Brazil \\ ${ }^{2}$ Veterinary Clinics Department, Sao Paulo State University, Sao Paulo, Brazil \\ ${ }^{3}$ Pathology Veterinary Department, Sao Paulo State University, Sao Paulo, Brazil \\ Email: erikaterra@ibest.com.br
}

Received December 16, 2011; revised January 4, 2012; accepted January 15, 2012

\begin{abstract}
Mammary tumors are very common in female dogs and its prognosis varies significantly according to the tumor invasion degree. Myoepithelial layer destruction is considered a differential diagnosis parameter of in situ from invasive lesions in human breast cancer. Aiming to evaluate the immunohistochemical expression of $\mathrm{p} 63$, a protein specifically expressed by myoepithelial cells of mammary gland, and its correlation with p53 expression, 10 adenomas and 20 carcinomas with and without metastasis of canine mammary gland were studied. There was no significant difference between adenomas and carcinomas for both proteins staining, however, the percentage and continuity of staining was lower in metastatic carcinomas. There was no correlation between p53 and p63 expression, but the higher values of p53 staining were found in carcinomas, according to previous studies. Results showed that p63 is specifically expressed also in myoepithelial cells of canine mammary gland. However, in order to ensure its use as a prognostic marker in canine mammary tumors, further studies involving a higher number of samples should be conducted.
\end{abstract}

Keywords: Bitches; Mammary Tumor; Prognostic Marker

\section{Introduction}

Mammary tumors are often diagnosed in bitches and are the most common type of tumors in veterinary routine. Veterinary clinicians and pathologists are frequently requested to provide a prognosis of their patients and metastasis and invasion are biological features of malignant tumors that have direct influence on treatment and prognosis of breast cancer patients [1-3].

During years, veterinary pathologists have focused on histopathological grade to determine the prognosis, however, with the disagreement between authors [4,5], the characterization of a prognosis through histopathological diagnosis have been discussed. Therefore prognostic factors with immunohistochemical markers have allowed additional criteria of malignancy to be evaluated [6].

p63 is a protein specifically expressed in breast myoepithelial cells. In women it is expressed in a decreasing manner from benign to malignant lesions and its expression is very low in invasive carcinomas, since one of the main criteria to classify an invasive carcinoma is the loss of myoepithelial cells $[7,8]$.

The TP53 gene is a tumor suppressor gene and the most frequently affected site of genetic alterations in human malignancies. It encodes the p53 protein, that controls the expression of a variety of genes involved in cell cycle regulation [9-11].

Aiming to investigate the immunohistochemical expression of $\mathrm{p} 63$ and its relation with p53, a study was performed with 10 adenomas and 20 simple and complex canine mammary carcinomas with and without metastasis.

\section{Materials and Methods}

The study protocol was approved by the Ethics Border on Animal Experimentation (CEEA). Thirty cases of canine mammary gland tumors with no breed or age predilection, were retrieved from the Department of Veterinary Pathology of São Paulo State University, Jaboticabal campus.

The animals were allotted in three groups:

(G1) 10 benign tumors;

(G2) 10 mammary carcinomas without metastasis at the diagnoses -5 complex carcinomas, 3 tubular carcinomas, 1 tubulopappilary carcinoma and 1 solid carcinoma;

(G3) 10 metastatic mammary carcinomas -6 tubulo- 
pappilary carcinomas, 3 solid carcinomas and 1 complex carcinoma.

All dogs were females, ranging from 3 to 15 years of age.

\subsection{Immunohistochemical Staining}

All tissue samples were routinely fixed in $10 \%$ neutral formalin and embedded in paraffin. Sections of $4 \mu \mathrm{m}$ were cut from one representative block of each case and collected onto glass slides. The slides were deparaffined, rehydrated in graded alcohols and submitted to heat-induced antigen retrieval with citrate $10 \mathrm{mM}$, pH 6.0 in water bath during 20 minutes for p63 and in pressure cooker (Pascal Dako) for p53. Endogenous peroxidase acitivity block was performed with $8 \%$ hydrogen peroxide in methanol.

Primary antibodies were diluted in phosphate-buffered saline and incubated for 60 minutes at $37^{\circ} \mathrm{C}$ (anti-p63) and for 18 hours at $4^{\circ} \mathrm{C}$ (anti-p53). Primary antibodies used for immunohistochemical evaluation included a monoclonal anti-human p63 (1:200, clone 4A4, Dako Cytomation, Carpinteria, CA, USA) and an anti-human polyclonal p53 antibody (1:500, clone CM1, Novocastra Laboratories, Newcastle, UK).

The secondary complex used was the EnVision (Dako). Diaminobenzidine (DAB) was used as a chromogen. Slides were counterstained with Mayer's hematoxilin, washed in running water for 5 minutes, dehydrated, and mounted in a synthetic medium. Adjacent normal tissue was used as positive control for p63 and canine mammary tumors previously known to express high levels of p53 were used as positive controls. Negative controls were obtained by omission of primary antibodies.

\subsection{Evaluation of Immunohistochemical Data and Statistical Analysis}

The number of p53 and p63 reactive cells was assessed semiquantitatively by two different pathologists using a score system: $0=$ no staining, $1=$ between 1 and $50 \%$ of stained cells (low staining) and $2=$ tumors with $>50 \%$ stained cells (high staining).

The comparisons between different groups and p53 and p63 expression were analyzed by Fisher's Exact test. To determine the correlation between antibodies, data was analyzed by Spearman's correlation test.

\section{Results and Discussion}

P63 staining was observed at the nuclei of mammary myoepithelial cells surrounding the alveoli and the staining decreased from benign to malignant tumors (Figure 1). There was also a decrease from tumors without metastasis to that with metastasis and this may indicate the destruction of myoepithelial cells layer as the lesion progresses. Staining absence was observed only in malignnant tumors with metastasis at the moment of diagnosis (Table 1). There was no significant differences between groups by Fisher's Exact Test $(\mathrm{p}<0.05)$.

When observed low percentage of p63 staining in breast cancer of women, some authors concluded that the p63 expression may be used as a differential diagnosis parameter between in situ and invasive lesions $[7,8]$. Similar results were found by another author in a study with mixed tumors from canine mammary gland [12].

Despite the absence of significance by Fisher's Exact test $(\mathrm{p}<0.05)$, it was observed that the percentage of $\mathrm{p} 53$ nuclear staining was higher between carcinomas. p53 staining has been demonstrated by several authors and it has always been identified in high quantities in malignant tumors [12-14]. However, p53 mutations should not be elected as unique events that initiate the carcinogenesis. The association between p53 and p63 was verified by Fischer's Exact Test which indicated that the variables are statistically independent $(\mathrm{p}>0.05)$. The Spearman Rank coefficient correlation between p53 and p63 shows that these two variables are statistically independents ( $p$ $<0.05)$.

\section{Conclusion}

In the present study p63 proved to be a specific marker for canine mammary myoepithelial cells, since there was no staining in other cell types of canine mammary gland. However there was no significant difference in this protein staining between adenomas and mammary carcinomas with and without metastasis (Fisher's Exact test, $\mathrm{p}<$ 0.05 ) and no correlation with p53 expression (Spearman's correlation test, $\mathrm{p}<0.05$ ). Thus, despite the fact of being a specific marker for canine mammary myoepithelial cells, p63 should not be used as the only prognostic marker, and further studies involving a larger number of animals must be conducted to certify its use.

\section{Acknowledgements}

This research was supported by Fundação de Apoio àPesquisa do Estado de São Paulo (FAPESP) and Coor-

Table 1. Percentage of negative, low $(+)$ and high (++) staining for $\mathrm{p} 63$ protein between different groups.

\begin{tabular}{cccc}
\hline \multirow{2}{*}{ Staining } & \multicolumn{3}{c}{ Groups } \\
\cline { 2 - 4 } & $\mathrm{G} 1$ & $\mathrm{G} 2$ & $\mathrm{G}^{\mathrm{a}}$ \\
\hline 0 & 0 & 0 & $10 \%$ \\
+ & $25 \%$ & $30 \%$ & $60 \%$ \\
++ & $75 \%$ & $70 \%$ & $30 \%$ \\
\hline
\end{tabular}

Primary tumors only. 


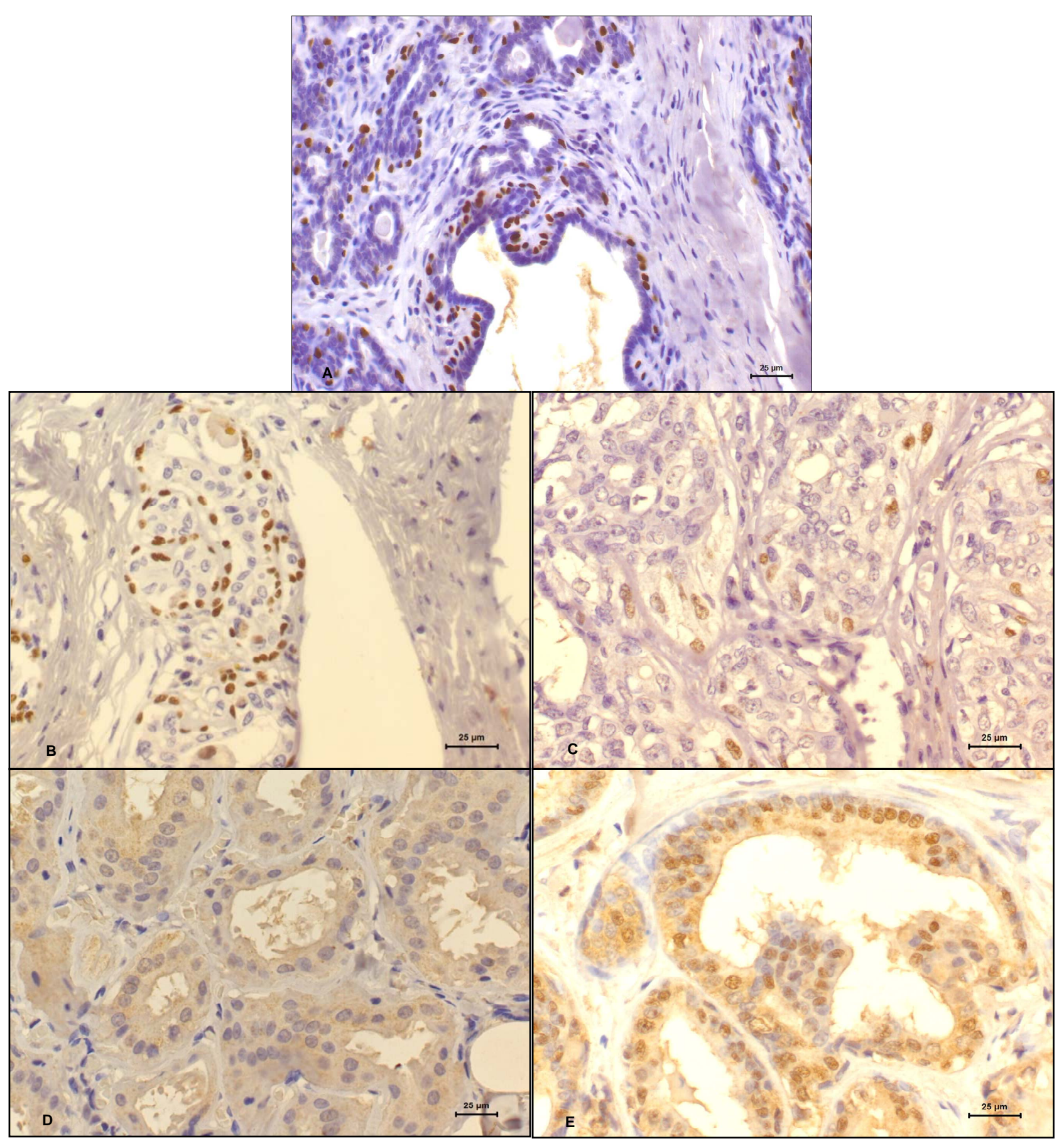

Figure 1. Adenoma (A), Non-metastatic tubular carcinoma (B) and Metastatic tubular carcinoma (C). See the loss of imunoreactivity and the discontinuous myoepithelial cell layers for (A) and (B) to (C). Immunohistochemistry, anti-p63, 400×; Adenoma (D) and Non-metastatic tubular carcinoma (E). See the no expression of p53 in the nucleus of epithelial cells in adenoma and the positivity of this protein in a carcinoma. Immunohistochemistry, anti-p53, 400×.

denação de Aperfeiçoamento de Pessoal de Nível Superior (CAPES).

\section{REFERENCES}

[1] C. R. Daleck, P. H. Franceschini, A. C. Alessi, A. E.
Santana and M. I. M. Martins, "Aspectos Clínico e Cirúrgico do Tumor Mamário Canino," Ciência Rural, Vol. 28, No. 1, 1998, pp. 95-100. doi:10.1590/S0103-84781998000100016

[2] S. Rodaski and C. H. Piekarz, "Epidemiologia e Etiologia do Câncer," In: C. R. Daleck, A. B. De Nardi and S. Rodaski, Eds., Oncologia em Cães e Gatos, Roca, São 
Paulo, 2008, pp. 1-22.

[3] B. G. Hollier, K. Evans and S. A. Mani, "The Epithelialto-Mesenchymal Transition and Cancer Stem Cells: A Coalition against Cancer Therapies," Journal of Mammary Gland Biology and Neoplasia, Vol. 14, No. 1, 2009, pp. 29-43. doi:10.1007/s10911-009-9110-3

[4] J. E. Moulton, "Tumors of the Mammary Gland," In: J. E. Moulton, Ed., Tumors in Domestic Animals, University of California Press, Berkeley, 1990, pp. 518-552.

[5] W. Misdorp, R. Else, E. Hellman, et al., "Histologic Classification of Mammary Tumors of the Dog and Cat," In: World Health Organization International Histological Classification of Tumors of Domestic Animals, Series 2, Vol. 7, No. 2, Armed Forces Institute of Pathology, Washington DC, 1999.

[6] J. Morris, "Improving the Diagnoses and Treatment of Canine Mammary Tumors: Immunohistochemical Markers as Prognostic Tools," The Veterinary Journal, Vol. 184, No. 1, 2010, pp. 3-4. doi:10.1016/j.tvj1.2009.07.003

[7] A. Ribeiro-Silva, L. N. Z. Ramalho, S. B. Garcia and S. Zucoloto, "The Relationship Between p63 and p53 Expression in Normal and Neoplastic Breast Tissue," Archives of Pathology \& Laboratory Medicine, Vol. 127, No. 3, 2003, pp. 336-340.

[8] D. Stefanou, A. Batistatou, A. Nonni, E. Arkoumani and N. J. Agnantis, "p63 Expression in Benign and Malignant Breast Lesions," Histology and Histopathology, Vol. 19,
No. 2, 2004, pp. 465-471.

[9] A. J. Levine, "p53, the Cellular Gatekeeper for Growth and Division," Cell, Vol. 88, No. 3, 1997, pp. 323-331.

[10] G. S. Goodsell, "The Molecular Perspective: p53 Tumor Suppressor," The Oncologist, Vol. 4, No. 2, 1999, pp. 138139.

[11] M. Levrero, V. De Laurenzi, A. Constanzo, S. Sabatini, J. Gong, J. Y. J. Wang, and G. Melino, "The p53/p63/p73 Family of Transcription Factors: Overlapping and Distinct Functions," Journal of Cell Science, Vol. 113, 2000, pp. 1661-1670.

[12] A. C. Bertagnolli, G. D. Cassali, M. C. L. S. Genelhu, F. A. Costa, J. F. C. Oliveira and P. B. D. Gonçalves, "Immunohistochemical Expression of p63 and $\Delta \mathrm{Np} 63$ in Mixed Tumors of Canine Mammary Glands and Its Relation with p53 Expression," Veterinary Pathology, Vol. 46, No. 3, 2009, pp. 407-415. doi:10.1354/vp.08-VP-0128-C-FL

[13] R. M. Gamblin, J. E. Sagartz and C. G. Couto, "Overexpression of p53 Tumor Suppressor Protein in Spontaneously Arising Neoplasms of Dogs," American Journal of Veterinary Research, Vol. 58, No. 8, 1997, pp. 857-863.

[14] C. H. Lee, W. H. Kim, J. H. Lim, M. S. Kang, D. Y. Kim and O. K. Kweon, "Mutation and Overexpression of p53 as a Prognostic Factor in Canine Mammary Tumors," Journal of Veterinary Science, Vol. 5, No. 1, 2004, pp. 6369. 